\title{
Effect of two-particle correlations on x-ray coherent diffractive imaging studies performed with continuum models
}

\author{
Zoltan Jurek, ${ }^{1,{ }^{*}}$ Robert Thiele, ${ }^{1}$ Beata Ziaja, ${ }^{1,2}$ and Robin Santra ${ }^{1,3}$ \\ ${ }^{1}$ Center for Free-Electron Laser Science, Deutsches Elektronen-Synchrotron, Notkestrasse 85, D-22607 Hamburg, Germany \\ ${ }^{2}$ Institute of Nuclear Physics, Polish Academy of Sciences, Radzikowskiego 152, 31-342 Krakow, Poland \\ ${ }^{3}$ Department of Physics, University of Hamburg, Jungiusstrasse 9, 20355 Hamburg, Germany \\ (Received 8 October 2011; revised manuscript received 7 May 2012; published 27 September 2012)
}

\begin{abstract}
Coherent diffraction imaging (CDI) of single molecules at atomic resolution is a major goal for the $\mathrm{x}$-ray free-electron lasers (XFELs). However, during an imaging pulse, the fast laser-induced ionization may strongly affect the recorded diffraction pattern of the irradiated sample. The radiation tolerance of the imaged molecule should then be investigated a priori with a dedicated simulation tool. The continuum approach is a powerful tool for modeling the evolution of irradiated large systems consisting of more than a few hundred thousand atoms. However, this method follows the evolution of average single-particle densities, and the experimentally recorded intensities reflect the spatial two-particle correlations. The information on these correlations is then inherently not accessible within the continuum approach. In this paper we analyze this limitation of continuum models and discuss the applicability of continuum models for imaging studies. We derive a formula to calculate scattered intensities (including both elastic and inelastic scattering) from the estimates obtained with a single-particle continuum model under conditions typical for CDI studies with XFELs. We demonstrate through numerical simulations that it describes the scattered signal with good accuracy. Two-particle correlation effects manifest themselves only in the region of low momentum transfers, together with the effects of the finite size of the sample. We also show that inelastic scattering on bound electrons can have a significant impact on the measured intensities: it contributes to the background that reduces the contrast of the recorded image. This effect is even more pronounced at larger momentum transfers. Therefore, whereas inelastic scattering can be neglected for nanocrystals, where Bragg scattering dominates, and in experiments imaging single objects at low resolution, it should be taken into account when planning atomic resolution imaging of nonperiodic samples. Finally, we show the effect of the electronic damage on the recorded total signal. Progressing damage does not change the positions of intensity peaks that correspond to the (fixed) positions of imaged ions. It only changes the contrast between intensity minima and maxima, which reduces the image contrast. Our results have implications for imaging-oriented studies of radiation damage performed with continuum models, as they define the limits of applicability of these models for CDI simulations.
\end{abstract}

DOI: 10.1103/PhysRevE.86.036411

PACS number(s): 52.65.-y, 61.05.cf, 41.60.Cr

\section{INTRODUCTION}

Coherent diffraction imaging (CDI) of single molecules at atomic resolution [1] is a major goal for the present and forthcoming $\mathrm{x}$-ray free-electron lasers (XFELs; LCLS, SACLA, and European XFEL) [2-4]. The high intensity and the extremely short pulse length $(<100 \mathrm{fs})$ of the XFEL beam are needed to get a sufficiently strong scattering signal from the sample and to reduce the effect of radiation damage (in comparison to low fluence imaging experiments, where the damage accumulates over many shots). The sample is destroyed during each measurement (so-called diffract-anddestroy method [1]). A single diffractive pattern is so noisy that averaging over patterns corresponding to the same molecular orientation is needed in order to increase the signal-to-noise ratio [5-10]. So for a three-dimensional (3D) reconstruction, many patterns of the same molecule are needed.

$\mathrm{X}$-ray diffraction gives information on the electron density of the system, so any changes of the electron density due to the

\footnotetext{
*Corresponding author: On leave from Wigner Research Centre for Physics, Institute for Solid State Physics and Optics, 1525 Budapest, P. O. Box 49, Hungary; zoltan.jurek@cfel.de
}

radiation damage influence the diffraction patterns. There are three main damage components: (i) atoms are "losing" bound electrons due to ionization, (ii) ionized atoms may move from their original positions, and (iii) scattering on the increasing free-electron density contributes to the signal. In order to investigate the effect of these damage components, detailed modeling and understanding of ionization dynamics are needed. The continuum approach (Boltzmann [11], hydrodynamic [12]) is an efficient way of modeling damage within large systems. However, presently available continuum methods follow the average single-particle densities, and the recorded diffraction intensities reflect two-particle correlations. The information on two-particle correlations is inherently not accessible from a single-particle continuum approach.

In this paper we analyze this limitation of continuum models and discuss the applicability of continuum models for imaging studies. In order to study this specific problem we do not need to construct a full $a b$ initio model of radiation damage. We analyze this problem with a simplified molecular dynamics model reproducing the conditions typical for coherent diffraction imaging studies that are known from experiments and from the theoretical studies of radiation damage [12]. The structure of this paper is as follows. In Sec. II we define the relation between the total (elastic and 
inelastic) diffracted signal and its corresponding estimate from the continuum model. In Sec. III we describe the physical conditions developing within XFEL irradiated samples. In Sec. IV we derive an approximate formula for signal scattered off an XFEL irradiated sample. It is derived from the estimates obtained with single-particle densities. This formula is then tested against numerical simulations in Sec. V. The results obtained are also discussed therein. Finally, in Sec. VI our conclusions are listed. The Appendix contains information on the simulation details.

\section{IMAGING STUDIES AND CONTINUUM MODELS}

The state of an imaged sample during a CDI experiment is dynamic; it changes with time as a consequence of the sample irradiation. Stochastically occurring ionizations change the electronic states of the atoms at different times, and the density of the released free electrons increases. Therefore the results of a CDI experiment, even when repeated under the same conditions, will vary due to the stochasticity. We call a unique time evolution that occurs during one CDI experiment a single realization.

In a single realization the total (elastic and inelastic) $\mathrm{x}$-ray intensity scattered off a system of electrons in state $|\Psi\rangle$ at time $t$ is proportional to $([13-15])$

$$
\begin{aligned}
I(\boldsymbol{q}, t) & =\int d^{3} r d^{3} r^{\prime}\left\langle\Psi\left|\hat{n}(\boldsymbol{r}, t) \hat{n}\left(\boldsymbol{r}^{\prime}, t\right)\right| \Psi\right\rangle e^{i \boldsymbol{q} \cdot\left(\boldsymbol{r}-\boldsymbol{r}^{\prime}\right)} \\
& =\left\langle\Psi\left|\hat{n}(\boldsymbol{q}, t) \hat{n}^{\star}(\boldsymbol{q}, t)\right| \Psi\right\rangle
\end{aligned}
$$

where $\hat{n}(\boldsymbol{r}, t)$ and $\hat{n}(\boldsymbol{q}, t)$ are the electron density operator and its Fourier transform, respectively,

$$
\begin{aligned}
& \hat{n}(\boldsymbol{r}, t)=\sum_{j=1}^{N_{e l}} \delta\left(\boldsymbol{r}-\hat{\boldsymbol{r}}_{j}(t)\right), \\
& \hat{n}(\boldsymbol{q}, t)=\int d^{3} r \hat{n}(\boldsymbol{r}, t) e^{i \boldsymbol{q} \cdot \boldsymbol{r}}=\sum_{j=1}^{N_{e l}} e^{i \boldsymbol{q} \cdot \hat{\boldsymbol{r}}_{j}(t)},
\end{aligned}
$$

and the operator $\hat{\boldsymbol{r}}_{j}(t)$ is the position operator in the Heisenberg picture.

Assuming the coherence time of the pulse to be short compared to the time scales of the processes occurring within the irradiated sample, the time integrated intensity can be approximated as an incoherent sum of the intensities scattered at instantaneous snapshots of the system. The number of photons scattered at the vector $\boldsymbol{q}$ during a single realization is then proportional to

$$
\mathscr{I}(\boldsymbol{q}) \equiv \int d t h(t) I(\boldsymbol{q}, t)
$$

The function $h(t)$ describes the average temporal envelope of the pulse, i.e., the modulus of the pulse amplitude squared, which is ensemble averaged over XFEL shots. During an imaging experiment a large number of patterns from single shots are collected and summed up in order to obtain an average pattern. Therefore the average of the recorded signal over the measured realizations $R$ must be formed:

$$
\langle\mathscr{I}(\boldsymbol{q})\rangle_{R}=\int d t h(t) \int d^{3} r d^{3} r^{\prime}\left\langle\hat{n}(\boldsymbol{r}, t) \hat{n}\left(\boldsymbol{r}^{\prime}, t\right)\right\rangle_{R} e^{i \boldsymbol{q} \cdot\left(\boldsymbol{r}-\boldsymbol{r}^{\prime}\right)} .
$$

For operators the symbol $\langle\cdots\rangle_{R}$ implies the averaging of the expectation value over realizations, i.e., for an observable $\hat{A}$ :

$$
\langle\hat{A}\rangle_{R} \equiv \sum p_{n}(t)\left\langle\Psi_{n}|\hat{A}| \Psi_{n}\right\rangle
$$

where $p_{n}(t)$ is the probability that the system is in one of the realized states $\left|\Psi_{n}\right\rangle$. For a classical quantity $B$ averaging over realization simplifies to

$$
\langle B\rangle_{R} \equiv \sum p_{n}(t) B_{n},
$$

where the quantity $B$ assumes a value $B_{n}$ with a probability $p_{n}(t)$ at time $t$. Here we would like to emphasize that our analysis is not restricted to systems in thermal equilibrium, so that the different realizations are not corresponding merely to the thermal fluctuations of an equilibrated system but may also lead through the nonequilibrium stages of the system evolution.

The continuum models, used to simulate the evolution of the irradiated systems, describe dynamical properties of electrons and ions, using average single-particle densities $\langle\hat{n}(\boldsymbol{r}, t)\rangle_{R}$. The intensity that one can construct directly from these average densities is

$$
\mathscr{I}^{C}(\boldsymbol{q})=\int d t h(t) \int d^{3} r d^{3} r^{\prime}\langle\hat{n}(\boldsymbol{r}, t)\rangle_{R}\left\langle\hat{n}\left(\boldsymbol{r}^{\prime}, t\right)\right\rangle_{R} e^{i \boldsymbol{q} \cdot\left(\boldsymbol{r}-\boldsymbol{r}^{\prime}\right)} .
$$

The difference between $\langle\mathscr{I}(\boldsymbol{q})\rangle_{R}$ and $\mathscr{I}^{C}(\boldsymbol{q})$ depends on twoparticle correlations during individual realizations.

\section{EVOLUTION OF X-RAY IRRADIATED BIOMOLECULES UNDER THE CONDITIONS TYPICAL FOR IMAGING STUDIES}

Detailed simulations of radiation damage $[1,12,16,17]$ reveal the typical ionization dynamics within an x-ray irradiated biological sample during an imaging experiment. Biological samples are typically built up from light elements such as $\mathrm{C}, \mathrm{N}$, $\mathrm{O}$, and H. Only a few atoms of heavy elements are present, e.g., $\mathrm{Cl}, \mathrm{S}$, and $\mathrm{Fe}$. The primary events that initiate radiation damage are the photoionization events [18]. Photoelectrons released by $\mathrm{x}$-rays from light elements have high energy $(\sim 10 \mathrm{keV})$. They can leave the irradiated system within a few femtoseconds, so that the net charge of the system starts to increase. During inner-shell photoionization, core holes are created. These holes are then filled by electrons from the outer shells within a few tens of femtoseconds. For light elements Auger decay is dominant [18]. Auger electrons have lower energies (about a few hundred eV), and they can be trapped within the charged sample. These quasifree electrons cause further ionizations that end up in secondary electron cascading $[19,20]$.

It is energetically preferred for the quasifree electrons to move towards the center of the sample, forming a net-neutral core with ions. This leads to the formation of a positively charged outer shell of ions around the neutral core. For samples large enough and sufficiently highly ionized, even high-energy 
photoelectrons can be trapped in the core, enhancing the temperature of the quasifree electrons.

Conditions of the electron plasma can be characterized by comparing the average potential energy of the electrostatic interaction between the electrons $E_{\text {Coulomb }}$ to their temperature $k_{B} T[21,22]$ :

$$
\Gamma=\frac{E_{\text {Coulomb }}}{k_{B} T}=\frac{1}{4 \pi \epsilon_{0}} \frac{e^{2}}{k_{B} T}\left(\frac{4 \pi n}{3}\right)^{\frac{1}{3}},
$$

where $n$ is the density of the electrons. In case of $\Gamma \gtrsim 1$, Coulomb interaction dominates the electron dynamics, and the plasma is strongly coupled. If $\Gamma \lesssim 1$, the Coulomb interaction is suppressed, and the plasma is weakly coupled.

Another parameter, the ratio of the electron temperature and the Fermi energy, $\Theta$, gives information on the quantum effects within the system [21,22]:

$$
\Theta=\frac{k_{B} T}{E_{F}}=\frac{k_{B} T}{\frac{\hbar^{2}}{2 m_{e}}\left(3 \pi^{2} n\right)^{2 / 3}} .
$$

At electron temperatures higher than the Fermi energy $(\Theta \gtrsim$ 1 ), a classical description is adequate, while at $\Theta \lesssim 1$, a quantum mechanical treatment is needed.

According to the aforementioned simulations [12], the typical electron density at the center of a biomolecule is around $10^{23} \mathrm{~cm}^{-3}$, and the electron temperature is $\sim 20 \mathrm{eV}$ when photoelectrons are not trapped and $\geqslant 100 \mathrm{eV}$ when photoelectrons are trapped. For the values of temperature $T=20 \mathrm{eV}$ and $T=100 \mathrm{eV}$, the plasma parameters are $\Gamma \approx 0.5, \Theta \approx 2.5$ and $\Gamma \approx 0.1, \Theta \approx 12.5$, respectively, so that the plasma of quasifree electrons can be treated as classical and ideal.

It should be noted that ions do not form a plasma during an imaging experiment. As the imaging pulses are short (approximately tens of femtoseconds) compared to electronion equilibration time (around a few picoseconds), ions remain "cold" during the pulse. Unscreened ions from the outer shell can move radially due to the repulsive Coulomb forces. However, if the pulse is not longer than several femtoseconds, those unscreened ions will also not relocate during the pulse $[12,23]$. As a result, during the imaging pulse, the initially neutral system evolves into a two-regime system consisting of "frozen" ions embedded in a thermalized free-electron plasma.

\section{IMPROVED DESCRIPTION OF DIFFRACTIVE SIGNAL OBTAINED FROM A SINGLE-PARTICLE CONTINUUM MODEL FOR A XFEL IRRADIATED SAMPLE}

In this section we investigate in detail how the scattered signal averaged over the realizations reflects the changing electron density within the irradiated sample. We follow the approach proposed by Chihara [24] and further applied in many works investigating x-ray scattering on plasmas (e.g., Refs. [25-28]). However, in our case we will apply it to a system that can be far from thermal equilibrium at its initial evolution stages. This is in contrast to the standard plasma applications that usually consider systems in thermal equilibrium.
Following [24], we separate the total electron density operator $\hat{n}(\boldsymbol{r}, t)$ into the bound electron component $\hat{n}_{b}(\boldsymbol{r}, t)$ and the unbound electron component. In general, one could develop a model for describing the dynamics of all electrons, including those that escape from the sample. However, the currently available continuum models [11,12] follow the dynamics of particles only within a restricted volume. Therefore we separate the unbound electron density operator into two components. One component refers to the electrons that escaped from the sample, $\hat{n}_{e}(\boldsymbol{r}, t)$, and the other one refers to the electrons trapped inside the sample, $\hat{n}_{t}(\boldsymbol{r}, t)$. From Eqs. (1) and (5) we then obtain

$$
\begin{aligned}
\langle I(\boldsymbol{q}, t)\rangle_{R}= & \left\langle\left|\int\left[\hat{n}_{b}(\boldsymbol{r}, t)+\hat{n}_{t}(\boldsymbol{r}, t)+\hat{n}_{e}(\boldsymbol{r}, t)\right] e^{i \boldsymbol{q} \cdot \boldsymbol{r}} d^{3} r\right|^{2}\right\rangle_{R} \\
= & \left\langle\left|\hat{n}_{b}(\boldsymbol{q}, t)\right|^{2}\right\rangle_{R}+\left\langle\left|\hat{n}_{t}(\boldsymbol{q}, t)\right|^{2}\right\rangle_{R}+\left\langle\left|\hat{n}_{e}(\boldsymbol{q}, t)\right|^{2}\right\rangle_{R} \\
& +2 \operatorname{Re}\left[\left\langle\hat{n}_{b}(\boldsymbol{q}, t) \hat{n}_{t}^{\star}(\boldsymbol{q}, t)\right\rangle_{R}+\left\langle\hat{n}_{b}(\boldsymbol{q}, t) \hat{n}_{e}^{\star}(\boldsymbol{q}, t)\right\rangle_{R}\right. \\
& \left.+\left\langle\hat{n}_{t}(\boldsymbol{q}, t) \hat{n}_{e}^{\star}(\boldsymbol{q}, t)\right\rangle_{R}\right] \\
= & \left\langle I_{b b}(\boldsymbol{q}, t)\right\rangle_{R}+\left\langle I_{t t}(\boldsymbol{q}, t)\right\rangle_{R}+\left\langle I_{e e}(\boldsymbol{q}, t)\right\rangle_{R} \\
& +2 \operatorname{Re}\left[\left\langle I_{b t}(\boldsymbol{q}, t)\right\rangle_{R}+\left\langle I_{b e}(\boldsymbol{q}, t)\right\rangle_{R}+\left\langle I_{t e}(\boldsymbol{q}, t)\right\rangle_{R}\right],
\end{aligned}
$$

where $I_{i j}(\boldsymbol{q}, t) \equiv\left\langle\Psi\left|\hat{n}_{i}(\boldsymbol{q}, t) \hat{n}_{j}^{\star}(\boldsymbol{q}, t)\right| \Psi\right\rangle$ for $i, j=b, t, e$. First, we analyze the effect of the diagonal terms, $\left\langle I_{b b}(\boldsymbol{q}, t)\right\rangle_{R}$, $\left\langle I_{t t}(\boldsymbol{q}, t)\right\rangle_{R}$, and $\left\langle I_{e e}(\boldsymbol{q}, t)\right\rangle_{R}$, and later, we analyze the effect of the cross terms, $\left\langle I_{b t}(\boldsymbol{q}, t)\right\rangle_{R},\left\langle I_{b e}(\boldsymbol{q}, t)\right\rangle_{R}$, and $\left\langle I_{t e}(\boldsymbol{q}, t)\right\rangle_{R}$.

\section{A. Bound electrons}

We can express the bound electron density operator as a convolution of atomic electron densities $\hat{n}_{b, j}(\boldsymbol{r}, t)$ and of the atomic position operators $\delta\left(\boldsymbol{r}-\hat{\boldsymbol{R}}_{j}(t)\right)[29]$ :

$$
\hat{n}_{b}(\boldsymbol{r}, t)=\sum_{j=1}^{N_{a}} \int d^{3} r^{\prime} \hat{n}_{b, j}\left(\boldsymbol{r}^{\prime}, t\right) \delta\left(\boldsymbol{r}-\boldsymbol{r}^{\prime}-\hat{\boldsymbol{R}}_{j}(t)\right) .
$$

Its Fourier transform then reads

$\hat{n}_{b}(\boldsymbol{q}, t)=\int \hat{n}_{b}(\boldsymbol{r}, t) e^{i \boldsymbol{q} \cdot \boldsymbol{r}} d^{3} r=\sum_{j=1}^{N_{a}} \hat{n}_{b, j}(\boldsymbol{q}, t) e^{i \boldsymbol{q} \cdot \hat{\boldsymbol{R}}_{j}(t)}$,

where $N_{a}$ is the total number of atoms, $\hat{\boldsymbol{R}}_{j}(t)$ is the position operator of atom $j$, and $\hat{n}_{b, j}(\boldsymbol{q}, t)$ is the Fourier transform of the density $\hat{n}_{b, j}(\boldsymbol{r}, t)$. The scattered intensity averaged over realizations is

$$
\begin{aligned}
\left\langle I_{b b}(\boldsymbol{q}, t)\right\rangle_{R} & =\left\langle\left|\hat{n}_{b}(\boldsymbol{q}, t)\right|^{2}\right\rangle_{R} \\
& =\sum_{j, k=1}^{N_{a}}\left\langle\hat{n}_{b, j}(\boldsymbol{q}, t) \hat{n}_{b, k}^{\star}(\boldsymbol{q}, t) e^{i \boldsymbol{q} \cdot\left(\hat{\boldsymbol{R}}_{j}(t)-\hat{\boldsymbol{R}}_{k}(t)\right)}\right\rangle_{R} .
\end{aligned}
$$

Radiation damage can affect the intensity pattern in two ways: (i) form factors of atoms can decrease due to the progressing ionization, and (ii) positions of ions can change due to the Coulomb interaction within the ionized system. The latter effect can be overcome by applying pulses short enough ( $\lesssim 10$ fs [23]), which are available at XFELs [30]. However, the reduction of the form factors cannot be eliminated. 
Assuming static atomic positions, $\left\langle\hat{\boldsymbol{R}}_{j}(t)\right\rangle_{R} \equiv \boldsymbol{R}_{j}$ in all realizations, and no correlation between ionization events of different atoms, so that $\left\langle\hat{n}_{b, j}(\boldsymbol{q}, t) \hat{n}_{b, k}^{\star}(\boldsymbol{q}, t)\right\rangle_{R}=\left\langle\hat{n}_{b, j}(\boldsymbol{q}, t)\right\rangle_{R}\left\langle\hat{n}_{b, k}^{\star}(\boldsymbol{q}, t)\right\rangle_{R}$ for $j \neq k$, the corresponding averaged intensity is

$$
\begin{aligned}
\left\langle I_{b b}^{\text {uncorr }}(\boldsymbol{q}, t)\right\rangle_{R} & =\sum_{j=1}^{N_{a}}\left\langle\left|\hat{n}_{b, j}(\boldsymbol{q}, t)\right|^{2}\right\rangle_{R}+\sum_{j \neq k=1}^{N_{a}}\left\langle\hat{n}_{b, j}(\boldsymbol{q}, t)\right\rangle_{R}\left\langle\hat{n}_{b, k}^{\star}(\boldsymbol{q}, t)\right\rangle_{R} e^{i \boldsymbol{q} \cdot\left(\boldsymbol{R}_{j}-\boldsymbol{R}_{k}\right)} \\
& =\sum_{j=1}^{N_{a}}\left\langle I_{j}^{\text {inel }}\right\rangle_{R}+\sum_{j=1}^{N_{a}}\left(\left\langle\left|f_{j}(\boldsymbol{q}, t)\right|^{2}\right\rangle_{R}-\left|\left\langle f_{j}(\boldsymbol{q}, t)\right\rangle_{R}\right|^{2}\right)+\left|\left\langle\sum_{j=1}^{N_{a}} f_{j}(\boldsymbol{q}, t) e^{i \boldsymbol{q} \cdot \boldsymbol{R}_{j}}\right\rangle_{R}\right|^{2} \\
& =\sum_{j=1}^{N_{a}} \frac{\left\langle N_{b, j}(t)\right\rangle_{R}}{Z_{j}} I_{\text {atomic }, Z_{j}}^{\text {inel }}+\sum_{j=1}^{N_{a}}\left(\left\langle\left|f_{j}(\boldsymbol{q}, t)\right|^{2}\right\rangle_{R}-\left|\left\langle f_{j}(\boldsymbol{q}, t)\right\rangle_{R}\right|^{2}\right)+\left|\left\langle n_{b}(\boldsymbol{q}, t)\right\rangle_{R}\right|^{2},
\end{aligned}
$$

where $f_{j}(\boldsymbol{q}, t) \equiv\left\langle\Psi\left|\hat{n}_{b, j}(\boldsymbol{q}, t)\right| \Psi\right\rangle$ is the atomic form factor and $n_{b}(\boldsymbol{q}, t) \equiv \sum_{j=1}^{N_{a}} f_{j}(\boldsymbol{q}, t) e^{i \boldsymbol{q} \cdot \boldsymbol{R}_{j}}$ is the classical bound electron density. Deriving the second line in Eq. (14), we used the following relation:

$$
\begin{aligned}
\left\langle\left|\hat{n}_{b, j}(\boldsymbol{q})\right|^{2}\right\rangle_{R} & =\sum_{n} p_{n}(t)\left(\left\langle\Psi_{n}\left|\hat{n}_{b, j}(\boldsymbol{q})\right| \Psi_{n}\right\rangle\left\langle\Psi_{n}\left|\hat{n}_{b, j}^{\star}(\boldsymbol{q})\right| \Psi_{n}\right\rangle+\left\langle\Psi_{n}\left|\hat{n}_{b, j}(\boldsymbol{q})\left(1-\left|\Psi_{n}\right\rangle\left\langle\Psi_{n}\right|\right) \hat{n}_{b, j}^{\star}(\boldsymbol{q})\right| \Psi_{n}\right\rangle\right) \\
& =\left\langle\left|f_{j}(\boldsymbol{q}, t)\right|^{2}\right\rangle_{R}+\left\langle I_{j}^{\text {inel }}\right\rangle_{R} .
\end{aligned}
$$

If the binding energy of a bound electron is much less than the energy of the incoming photon, the intensity $I_{j}^{\text {inel }}$ scattered inelastically on the atom or ion $j$ can be approximated as being proportional to the number of its bound electrons $N_{b, j}(t)$ multiplied by the intensity $I_{\text {atomic }, Z_{j}}^{\text {inel }}$ scattered inelastically from the neutral element of atomic number $Z_{j}$ :

$$
\left\langle I_{j}^{\text {inel }}\right\rangle_{R}=\frac{\left\langle N_{b, j}(t)\right\rangle_{R}}{Z_{j}} I_{\text {atomic }, Z_{j}}^{\text {inel }}
$$

According to Eq. (14) (last line), the total averaged scattered intensity from bound electrons is then the sum of (i) the inelastic incoherent scattering component $\sum_{j=1}^{N_{a}}\left\langle I_{j}^{\text {inel }}\right\rangle_{R}$, (ii) the elastic incoherent scattering component containing the variance of the atomic form factors of the individual ions, and (iii) the density $\left|\left\langle n_{b}(\boldsymbol{q}, t)\right\rangle_{R}\right|^{2}$ that describes coherent elastic scattering. Similar terms appear in the final formula of Ref. [24], except for the term which is a consequence of the externally driven ionization dynamics.

\section{B. Trapped electrons}

We investigate now the contribution of trapped electrons to the imaging pattern. Under conditions typical for CDI studies electrons can be treated as classical particles. In what follows we will then replace the electron-density-operator expectation values $\langle\Psi|\hat{n}(\boldsymbol{r}, t)| \Psi\rangle$ with their corresponding classical equivalents $n(\boldsymbol{r}, t)$. The classical electron density and its Fourier transform, defined for $N_{t}$ trapped electrons, located at positions $\boldsymbol{r}_{j}(t)$ at time $t$, then are

$$
\begin{aligned}
& n_{t}(\boldsymbol{r}, t)=\sum_{j=1}^{N_{t}} \delta\left(\boldsymbol{r}-\boldsymbol{r}_{j}(t)\right), \\
& n_{t}(\boldsymbol{q}, t)=\int d^{3} r n_{t}(\boldsymbol{r}, t) e^{i \boldsymbol{q} \cdot \boldsymbol{r}}=\sum_{j=1}^{N_{t}} e^{i \boldsymbol{q} \cdot \boldsymbol{r}_{j}(t)},
\end{aligned}
$$

and the realization-averaged signal scattered from trapped electrons is proportional to

$$
\left\langle I_{t t}(\boldsymbol{q}, t)\right\rangle_{R}=N_{t}+\sum_{j \neq k=1}^{N_{t}}\left\langle e^{i \boldsymbol{q} \cdot\left[r_{j}(t)-\boldsymbol{r}_{k}(t)\right]}\right\rangle_{R} .
$$

Using the two-particle distribution function [31], one can rewrite it further as

$$
\begin{aligned}
\left\langle I_{t t}(\boldsymbol{q}, t)\right\rangle_{R}= & N_{t}+\int d^{3} r d^{3} r^{\prime}\left\langle n_{t}(\boldsymbol{r}, t)\right\rangle_{R}\left\langle n_{t}\left(\boldsymbol{r}^{\prime}, t\right)\right\rangle_{R} \\
& \times g\left(\boldsymbol{r}, \boldsymbol{r}^{\prime}, t\right) e^{i \boldsymbol{q} \cdot\left(\boldsymbol{r}-\boldsymbol{r}^{\prime}\right)} \\
= & N_{t}+\frac{N_{t}-1}{N_{t}}\left|\left\langle n_{t}(\boldsymbol{q}, t)\right\rangle_{R}\right|^{2} \\
& +\int d^{3} r d^{3} r^{\prime}\left\langle n_{t}(\boldsymbol{r}, t)\right\rangle_{R}\left\langle n_{t}\left(\boldsymbol{r}^{\prime}, t\right)\right\rangle_{R} \\
& \times\left[g\left(\boldsymbol{r}, \boldsymbol{r}^{\prime}, t\right)-\frac{N_{t}-1}{N_{t}}\right] e^{i \boldsymbol{q} \cdot\left(\boldsymbol{r}-\boldsymbol{r}^{\prime}\right)},
\end{aligned}
$$

where $g\left(\boldsymbol{r}, \boldsymbol{r}^{\prime}, t\right)$ is the two-particle distribution function at time $t$. The time dependence of $g\left(\boldsymbol{r}, \boldsymbol{r}^{\prime}, t\right)$ is imposed by the nonequilibrium evolution of the considered system.

In the second line of Eq. (19) the first term is a $q$ independent shift given by the number of trapped electrons $N_{t}$. The second term depends on the average electron density. The third term contains the statistical information about the two-particle correlations. This information is not accessible within the single-particle continuum approach, as considered here. The first and third terms correspond to the incoherent scattering, whereas the second one corresponds to coherent scattering on free electrons as in Chihara's formula in Ref. [24].

Neglecting all correlations in Eq. (19), we arrive at the following estimate:

$$
\left\langle I_{t t}^{\mathrm{uncorr}}(\boldsymbol{q}, t)\right\rangle_{R}=N_{t}+\frac{N_{t}-1}{N_{t}}\left|\left\langle n_{t}(\boldsymbol{q}, t)\right\rangle_{R}\right|^{2} .
$$

In the above derivations we assumed that the number of trapped electrons $N_{t}$ is identical for all realizations at any time 
$t$. As the $N_{t}$ distribution results from a stochastic process, this assumption is generally not valid. In the general case, we should replace $N_{t}$ with its average over realizations at time $t$, $\left\langle N_{t}(t)\right\rangle_{R}$. This is an accurate approximation if the number of particles in individual realizations is large. For a small number of particles this may introduce a large relative error. However, in our case, the contribution of trapped electrons to the overall scattered intensity is small, so in any case it should not introduce a significant error.

We note that the approximation, Eq. (20), could be further improved by taking into account correlations. In general, especially in case of short pulses, the electrons may not reach thermal equilibrium during the pulse. This would require a time-dependent parametrization of $g\left(\boldsymbol{r}, \boldsymbol{r}^{\prime}, t\right)$. If the pulse is long enough compared to the electron thermalization time scale, the two-particle distribution function for electrons could be approximated by the equilibrium one.

\section{Escaped electrons}

Energetic photoelectrons leave the sample soon after exposure to the $\mathrm{x}$-ray pulse starts. However, they may stay within the focus of the beam during the imaging pulse and also contribute to the diffractive patterns.

In order to describe their contribution to the intensity, the same approximate formula as for the trapped electrons, Eq. (20), can be applied:

$$
\left\langle I_{e e}^{\text {uncorr }}(\boldsymbol{q}, t)\right\rangle_{R}=N_{e}+\frac{N_{e}-1}{N_{e}}\left|\left\langle n_{e}(\boldsymbol{q}, t)\right\rangle_{R}\right|^{2} .
$$

In the case of escaped electrons, their density is dilute; e.g., the distances between escaped electrons are on average large. Their contribution will then show up mainly at small values of $q$, limited by the inverse of the size of the escaped electron cloud. We explain here that the relevant $q$ range for imaging studies is defined by $1 / L<q / 2 \pi<2 / d$, where $L$ is the size of the object (here the size of the electron cloud) and $d$ is the desired resolution. Therefore, if the continuum model does not provide information on $\left\langle n_{e}(\boldsymbol{q}, t)\right\rangle_{R}$, we can approximate the effect of the escaped electrons as

$$
\left\langle I_{e e}^{\mathrm{uncorr}}(\boldsymbol{q}, t)\right\rangle_{R} \cong N_{e},
$$

which is accurate enough in the high $q$ regime, limited by the inverse resolution, $2 / d$.

\section{Cross terms}

The escaping high-energy electrons are typically located at large distances from other particles, so the potential energy of their individual interaction with other particles is much smaller than their kinetic energy. Therefore, the Coulomb correlations between bound electrons and escaped electrons and between trapped electrons and escaped electrons can be neglected, and the respective cross terms factorize to

$$
\begin{aligned}
\left\langle I_{b e}(\boldsymbol{q}, t)\right\rangle_{R} & =\left\langle n_{b}(\boldsymbol{q}, t) n_{e}^{\star}(\boldsymbol{q}, t)\right\rangle_{R}=\left\langle n_{b}(\boldsymbol{q}, t)\right\rangle_{R}\left\langle n_{e}^{\star}(\boldsymbol{q}, t)\right\rangle_{R}, \\
\left\langle I_{t e}(\boldsymbol{q}, t)\right\rangle_{R} & =\left\langle n_{t}(\boldsymbol{q}, t) n_{e}^{\star}(\boldsymbol{q}, t)\right\rangle_{R}=\left\langle n_{t}(\boldsymbol{q}, t)\right\rangle_{R}\left\langle n_{e}^{\star}(\boldsymbol{q}, t)\right\rangle_{R} .
\end{aligned}
$$

Following our discussion from the previous section, we can neglect these terms in the considered high $q$ regime.
The correlation between bound and trapped electrons, originating from the same initial process of ionization, strongly decreases with time as the number of particles increases and the particle cloud evolves. However, there are still Coulomb correlations between these particles. If we compare the potential energy of the electrons trapped in the field of the ions to their kinetic energy at the typical simulation parameters [12], we obtain

$$
\begin{aligned}
& \Gamma_{e i} \approx 0.93 \quad \text { at } \quad T=20 \mathrm{eV}, \\
& \Gamma_{e i} \approx 0.18 \quad \text { at } \quad T=100 \mathrm{eV} .
\end{aligned}
$$

If the electron-ion system is weakly coupled $\left(\Gamma_{e i}<1\right)$, we can then neglect the correlations between the different density terms, and the cross term can be factorized as

$$
\left\langle I_{b t}(\boldsymbol{q}, t)\right\rangle_{R}=\left\langle n_{b}(\boldsymbol{q}, t) n_{t}^{\star}(\boldsymbol{q}, t)\right\rangle=\left\langle n_{b}(\boldsymbol{q}, t)\right\rangle_{R}\left\langle n_{t}^{\star}(\boldsymbol{q}, t)\right\rangle_{R} .
$$

Again, the cross terms then depend solely on average densities, which are known from the continuum model. A similar cross term appears in Chihara's formula in Ref. [24].

\section{E. Intensities from the continuum model}

Here we collect the terms that we derived in the previous sections. The estimate for the total scattered signal in the uncorrelated case in the region of high $q$ values is

$$
\begin{aligned}
\left\langle I^{\text {uncorr }}(\boldsymbol{q}, t)\right\rangle_{R} & \sum_{j=1}^{N_{a}} \frac{\left\langle N_{b, j}(t)\right\rangle_{R}}{Z_{j}} I_{\text {atomic }, Z_{j}}^{\text {inel }}+\sum_{j=1}^{N_{a}}\left(\left\langle\left|f_{j}(\boldsymbol{q}, t)\right|^{2}\right\rangle_{R}\right. \\
= & \left.-\left|\left\langle f_{j}(\boldsymbol{q}, t)\right\rangle_{R}\right|^{2}\right)+\left|\left\langle n_{b}(\boldsymbol{q}, t)\right\rangle_{R}\right|^{2}+N_{t} \\
& +\frac{N_{t}-1}{N_{t}}\left|\left\langle n_{t}(\boldsymbol{q}, t)\right\rangle_{R}\right|^{2}+N_{e} \\
& +2 \operatorname{Re}\left[\left\langle n_{b}(\boldsymbol{q}, t)\right\rangle_{R}\left\langle n_{t}^{\star}(\boldsymbol{q}, t)\right\rangle_{R}\right] \\
= & I^{C}(\boldsymbol{q}, t)+\sum_{j=1}^{N_{a}}\left(\left\langle\left|f_{j}(\boldsymbol{q}, t)\right|^{2}\right\rangle_{R}-\left|\left\langle f_{j}(\boldsymbol{q}, t)\right\rangle_{R}\right|^{2}\right) \\
& +\sum_{j=1}^{N_{a}} \frac{\left\langle N_{b, j}(t)\right\rangle_{R}}{Z_{j}} I_{\text {atomic }, Z_{j}}^{\text {inel }}-\frac{1}{N_{t}} I_{t}^{C}(\boldsymbol{q}, t)+N_{t}+N_{e},
\end{aligned}
$$

where $I^{C}$ is the intensity obtained from the total average electron density within the continuum model [cf. Eq. (7)]:

$$
\begin{aligned}
I^{C}(\boldsymbol{q}, t) & =\left|\left\langle n_{b}(\boldsymbol{q}, t)\right\rangle_{R}+\left\langle n_{t}(\boldsymbol{q}, t)\right\rangle_{R}\right|^{2} \\
& =I_{b}^{C}(\boldsymbol{q}, t)+I_{t}^{C}(\boldsymbol{q}, t)+2 \operatorname{Re}\left[\left\langle n_{b}(\boldsymbol{q}, t)\right\rangle_{R}\left\langle n_{t}^{\star}(\boldsymbol{q}, t)\right\rangle_{R}\right],
\end{aligned}
$$

and $I_{b, t}^{C}(\boldsymbol{q}, t) \equiv\left|\left\langle n_{b, t}(\boldsymbol{q}, t)\right\rangle_{R}\right|^{2}$. If the correlations within the electronic system are known, Eq. (26) can be improved by including Eq. (19). Otherwise, the uncorrelated approximation, Eq. (20), should be used.

\section{COMPARISON TO NUMERICAL SIMULATIONS}

In order to investigate the applicability regime of Eq. (26), we performed dedicated molecular dynamics (MD) 
simulations of electron-ion systems at the conditions corresponding to those that develop in the center of irradiated biological samples during an imaging experiment. For technical details, see the Appendix.

Using MD simulations, one can follow individual realizations of the dynamics within an irradiated system. This enables a direct comparison of the time-integrated realizationaveraged diffraction signal to its estimate, Eq. (26), derived from single-particle densities. It corresponds to the estimate that could be obtained from a continuum model. From the results of MD simulations we calculated (i) the average intensity from different realizations $\langle I(\boldsymbol{q}, t)\rangle_{R}$ [Eqs. (3) and (4)], (ii) the uncorrelated intensity $\left\langle I^{\text {uncorr }}(\boldsymbol{q}, t)\right\rangle_{R}$ [Eq. (26)], and (iii) the correlated intensity $\left\langle I^{\text {corr }}(\boldsymbol{q}, t)\right\rangle_{R}[\mathrm{Eq}$. (19), for electrons only]. We integrated them over time to obtain $\langle\mathscr{I}(\boldsymbol{q})\rangle_{R},\left\langle\mathscr{I}^{\text {uncorr }}(\boldsymbol{q})\right\rangle_{R}$, and $\left\langle\mathscr{I}^{\text {corr }}(\boldsymbol{q})\right\rangle_{R}$, respectively. We also calculated $\mathscr{I}^{C}(\boldsymbol{q})$, according to Eq. (7). We investigated the following cases.

Scattering from bound electrons only. We modeled a cluster consisting of 100 initially neutral carbon atoms. The atoms were located randomly within a sphere of radius $R_{0}=7.1 \AA$. Their density was $1 / 15 \AA^{-3}$, which corresponds to a typical protein density. The nearest neighbor distances were always larger than $1.5 \AA$. The atoms were not moving. The time dependence of the average number of bound electrons per ion was approximated by an exponential function in such a way that the average number of bound electrons per atom decreased from 6 to 4 during the simulation time.

Scattering from trapped electrons only. Electrons were treated as classical particles with regularized Coulomb interaction between them. The granular ions were not included. However, an average positive field (radial harmonic potential) was added to keep the electrons together. The strength of the harmonic potential was determined by the average ion density and the average ion charge. The electron dynamics was followed by integrating the equations of motion of the electrons. The parameters we used corresponded to the results of the damage modeling for biosamples [12]: $n_{\text {atom }}=1 / 15 \AA^{-3}$ (average atomic density), $Q_{\text {ion }}=2$ (average ion charge). The number of electrons was $N=200$. The electrons filled a sphere of $R_{0}=7.1 \AA \AA$ radius at a density of $2 n_{\text {atom }}$. We followed 100 realizations of the system during $T_{\text {pulse }}=10 \mathrm{fs}$, from which we calculated the realization average. We performed two sets of calculations, one with an electron temperature of $20 \mathrm{eV}$ and another one with $T_{e}=100 \mathrm{eV}$, which corresponded to the cases when photoelectrons could escape or were trapped within the system, respectively.

Scattering from bound and trapped electrons. This case was just the merging of the above two cases, with the same values of the simulation parameters. A regularized Coulomb potential described the interaction between granular ions and electrons. The external harmonic potential was not applied here. Below we discuss the simulation results that we have obtained.

\section{A. Bound electrons}

The scattered intensity depends on stochastic properties of the ionization process. Figure 1 shows the fraction of ions with different charges created within the sample. Although we used a simplified model for describing the ionization (see

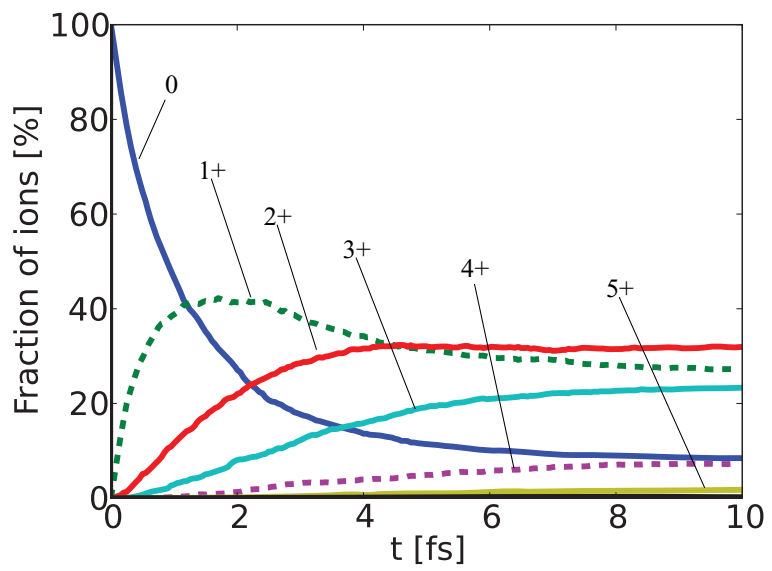

FIG. 1. (Color online) Average ion-charge-state populations within the imaged system as a function of time.

the Appendix), the trends in the charge distributions are in agreement with Ref. [12].

In Fig. 2 we show the intensity distribution for scattering from bound electrons along a randomly chosen axis in reciprocal space. The parameter $\left\langle\mathscr{I}_{b b}^{\text {uncorr }}(\boldsymbol{q})\right\rangle_{R}$ gives a good estimate of $\left\langle\mathscr{I}_{b b}(\boldsymbol{q})\right\rangle_{R}$. There is a disagreement between $\mathscr{I}_{b}^{C}(\boldsymbol{q})$ and $\left\langle\mathscr{I}_{b b}(\boldsymbol{q})\right\rangle_{R}$, as there is no inelastic scattering included in $\mathscr{I}_{b}^{C}(\boldsymbol{q})$. The shift between these two intensities is proportional to the number of bound electrons at high $q$. Therefore, one should instead use here $\left\langle\mathscr{I}_{b b}^{\text {uncorr }}(\boldsymbol{q})\right\rangle_{R}$ as an estimate of the scattered intensity from bound electrons.

\section{B. Trapped electrons}

The electronic system considered here is in thermal equilibrium. The inverse Debye length,

$$
k_{D} \equiv \sqrt{\frac{n_{t} e^{2}}{\epsilon_{0} k_{B} T}},
$$

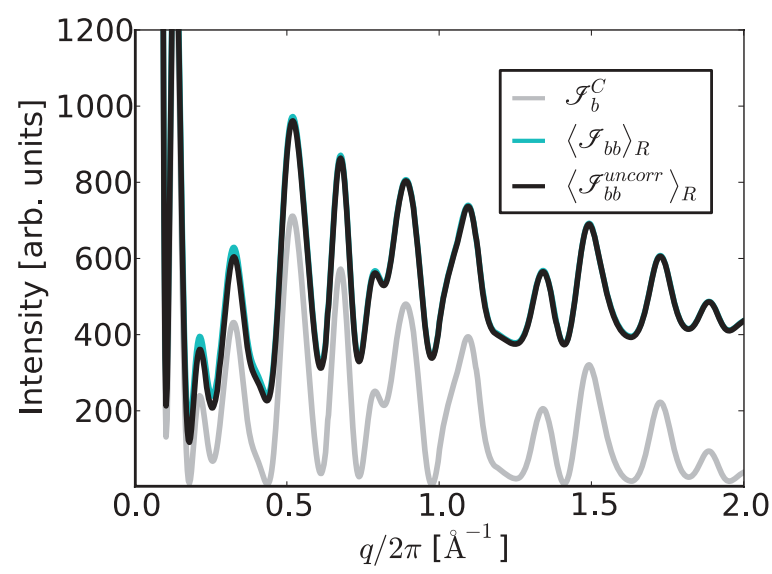

FIG. 2. (Color online) Time integrated intensity from diffractive scattering only by bound electrons as a function of the momentum transfer, $q$, along a random axis in reciprocal space. We show (i) the intensity constructed from average single particle densities $\mathscr{I}_{b}^{C}$, (ii) the scattered intensity $\left\langle\mathscr{I}_{b b}\right\rangle_{R}$, and (iii) the estimated uncorrelated intensity $\left\langle\mathscr{I}_{b b}^{\text {uncorr }}\right\rangle_{R}$. The curves (ii) and (iii) almost overlap. 


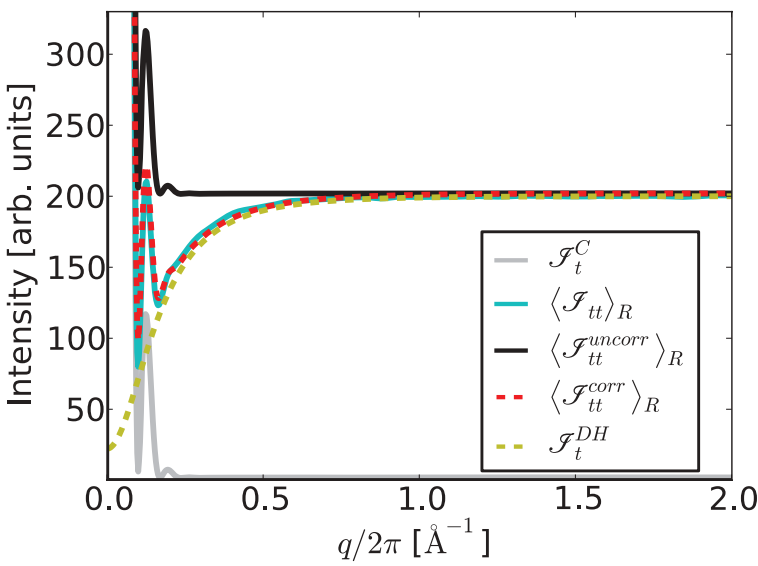

FIG. 3. (Color online) Time integrated intensity from diffractive scattering by trapped electrons as a function of the momentum transfer $q$. The electron temperature was set to $20 \mathrm{eV}$, and an external harmonic potential was applied. We show (i) the intensity constructed from average single-particle densities $\mathscr{I}_{t}^{C}$, (ii) the scattered intensity $\left\langle\mathscr{I}_{t t}\right\rangle_{R}$, (iii) the estimated uncorrelated intensity $\left\langle\mathscr{I}_{t t}^{\text {uncorr }}\right\rangle_{R}$, (iv) the estimated correlated intensity $\left\langle\mathscr{I}_{t t}^{\text {corr }}\right\rangle_{R}$, and (v) the intensity obtained from the theory of homogeneous, infinite-size, weakly coupled plasmas $\mathscr{I}_{t}^{\mathrm{DH}}$.

is $\approx 1 \AA^{-1}$ at an electron temperature of $20 \mathrm{eV}$. In Fig. 3, we show the time integrated scattered intensity $\left\langle\mathscr{I}_{t t}(\boldsymbol{q})\right\rangle_{R}$ along an axis in reciprocal space, averaged over realizations, as compared to the intensity obtained from the average density $\mathscr{I}_{t}^{C}(\boldsymbol{q})$, and to the intensity estimated in the uncorrelated case $\left\langle\mathscr{I}_{t t}^{\text {uncorr }}(\boldsymbol{q})\right\rangle_{R}$. The dominant correction [to $\mathscr{I}_{t}^{C}(\boldsymbol{q})$ ] originates from the granularity of electrons and is given by the total number of the trapped electrons in the system [cf. Eq. (20)]. At larger momentum transfers, $q>k_{D}$, this correction is accurate enough and $\left\langle\mathscr{I}_{t t}^{\text {uncorr }}(\boldsymbol{q})\right\rangle_{R}$ agrees well with $\left\langle\mathscr{I}_{t t}(\boldsymbol{q})\right\rangle_{R}$. However, at lower momentum transfer, it breaks down. The reason for this is that in Eq. (20) all correlations are neglected a priori, and in our simulated case two-particle correlations are present. They manifest themselves at low $q$. As expected, their effect decreases at higher electron temperatures, $T=100 \mathrm{eV}$ (Fig. 4).

For an infinite, homogeneous system, the scattered intensity is connected to the radial distribution function by the following expression [31]:

$$
\mathscr{I}(\boldsymbol{q})=N_{t}\left[1+n_{t} \int d^{3} r(g(\boldsymbol{r})-1) e^{i \boldsymbol{q} \cdot \boldsymbol{r}}\right],
$$

where $n_{t}$ is the homogeneous density of the electrons. In the case of a Debye-Hückel plasma, the radial distribution function $g(r)$ is [31]

$$
g^{\mathrm{DH}}(r)=\exp \left(-\frac{k_{D}^{2}}{4 \pi n_{t} r} e^{-k_{D} r}\right) .
$$

Apart from the region of $q \lesssim 1 / R_{0}$, where the finite-size effects dominate, at $T=20 \mathrm{eV}\left\langle\mathscr{I}_{t t}(\boldsymbol{q})\right\rangle_{R}$ remains in good agreement with the intensity profile $\mathscr{I}_{t}^{\mathrm{DH}}(\boldsymbol{q})$ obtained from Eqs. (28) and (29), evaluated with the initial simulation parameters, $R_{0}=7.1 \AA$, $n_{t}=N_{t} /\left(4 \pi R_{0}^{3} / 3\right)$. A better estimate, $\left\langle\mathscr{I}_{t t}^{\text {corr }}(\boldsymbol{q})\right\rangle_{R}$, can be obtained by evaluating Eq. (19) with the

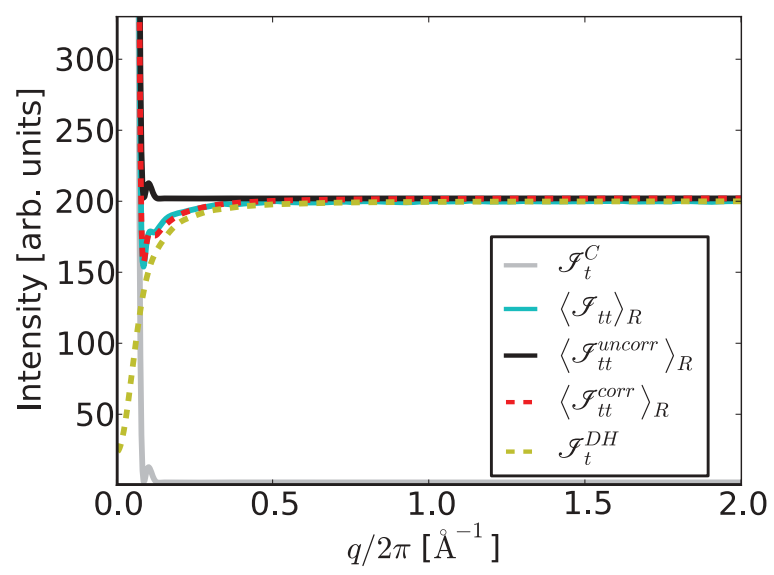

FIG. 4. (Color online) Time integrated intensity from diffractive scattering by trapped electrons as a function of the momentum transfer $q$. The electron temperature was set to $100 \mathrm{eV}$, and an external harmonic potential was applied. We show (i) the intensity constructed from average single-particle densities $\mathscr{I}_{t}^{C}$, (ii) the scattered intensity $\left\langle\mathscr{I}_{t t}\right\rangle_{R}$, (iii) the estimated uncorrelated intensity $\left\langle\mathscr{I}_{t t}^{\text {uncorr }}\right\rangle_{R}$, (iv) the estimated correlated intensity $\left\langle\mathscr{I}_{t t}^{\text {corr }}\right\rangle_{R}$, and (v) the intensity obtained from the theory of homogeneous, infinite-size, weakly coupled plasmas $\mathscr{I}_{t}^{\mathrm{DH}}$.

pair correlation function, Eq. (30). It then applies well in the entire $q$ range.

At the higher temperature $(T=100 \mathrm{eV})$, the spread of the electron density is wider (Fig. 5). We then have to evaluate Eqs. (19) and (28)-(30) with parameters that correspond to a homogeneous sphere with the average radius estimated to be $R \approx 8.2 \AA$. Our findings are similar: the intensity fit $\left\langle\mathscr{I}_{t t}^{\text {corr }}(\boldsymbol{q})\right\rangle_{R}$ obtained from Eq. (19) is in good agreement with the total signal. To sum up, $\left\langle\mathscr{I}_{t t}^{\text {uncorr }}(\boldsymbol{q})\right\rangle_{R}$ overestimates the full scattered intensity at $q \lesssim k_{D}$; however, it still gives an accurate estimate of the trapped-electron background in the

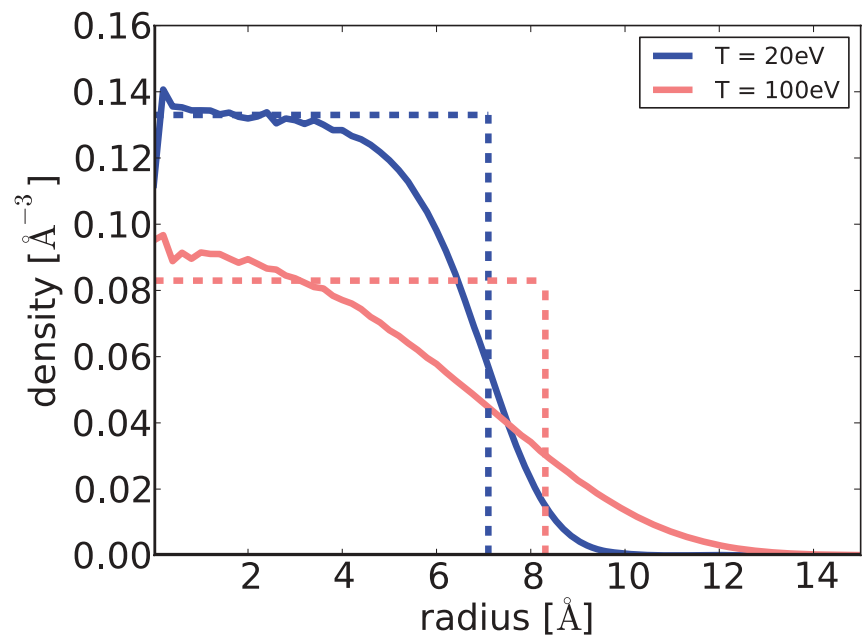

FIG. 5. (Color online) Average density of an electron system containing 200 electrons (solid lines) at temperatures $20 \mathrm{eV}$ and $100 \mathrm{eV}$, with an external harmonic potential applied. Dashed lines show the radius and density values used for evaluating Eqs. (19) and (30). 


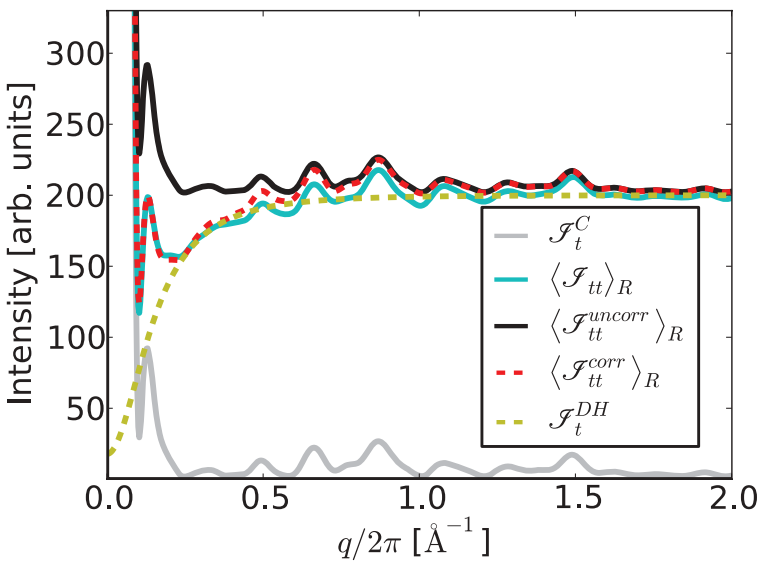

FIG. 6. (Color online) Time integrated intensity from diffractive scattering by trapped electrons as a function of the momentum transfer $q$. The electrons were moving in the field of positive granular ions. The electron temperature was set to $20 \mathrm{eV}$. We show (i) the intensity constructed from average single-particle densities $\mathscr{I}_{t}^{C}$, (ii) the scattered intensity $\left\langle\mathscr{I}_{t t}\right\rangle_{R}$, (iii) the estimated uncorrelated intensity $\left\langle\mathscr{I}_{t t}^{\text {uncorr }}\right\rangle_{R}$, (iv) the estimated correlated intensity $\left\langle\mathscr{I}_{t t}^{\text {corr }}\right\rangle_{R}$, and (v) the intensity obtained from the Debye-Hückel theory $\mathscr{I}_{t}^{\mathrm{DH}}$.

region of high $q$, important for high resolution imaging. The estimate $\left\langle\mathscr{I}_{t t}^{\text {corr }}(\boldsymbol{q})\right\rangle_{R}$ applies well in the entire $q$ region.

\section{Bound and trapped electrons}

Finally, we discuss the case with contributions from both bound and trapped electrons. In this simulation electrons were moving in the field of granular ions; therefore we can expect some change in the behavior of the average intensity scattered by the trapped electrons (Fig. 6) as compared to that scattered by the trapped electrons in a smooth positive field (Fig. 3). Discrepancies show up as small peaks at the $q$ values

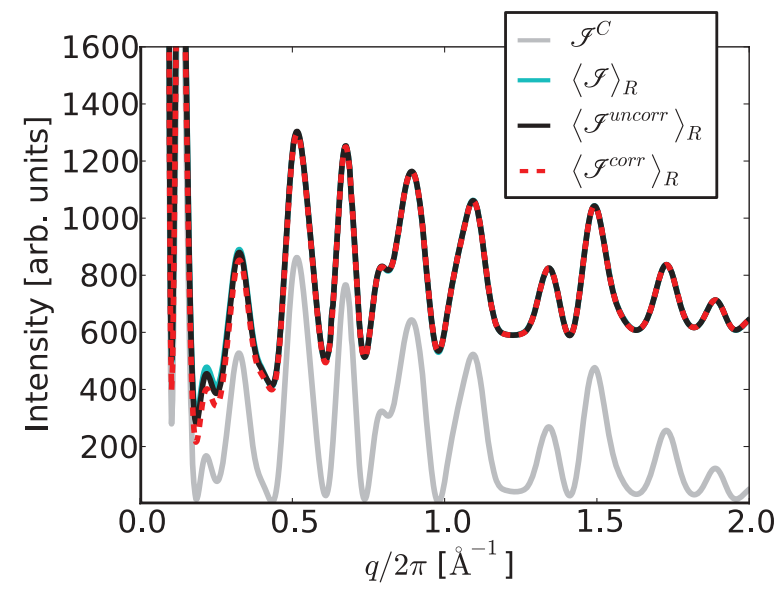

FIG. 7. (Color online) Time integrated intensity from diffractive scattering by bound and trapped electrons as a function of the momentum transfer $q$ along a random axis in reciprocal space. We show (i) the intensity constructed from average single-particle densities $\mathscr{I}^{C}$, (ii) the full scattered intensity $\langle\mathscr{I}\rangle_{R}$, (iii) the estimated uncorrelated intensity $\left\langle\mathscr{I}^{\text {uncorr }}\right\rangle_{R}$, and (iv) the estimated correlated intensity $\left\langle\mathscr{I}_{t t}^{\text {corr }}\right\rangle_{R}$. The curves for intensities (ii), (iii), and (iv) almost overlap. corresponding to the locations of ion peaks (Fig. 2). These peaks are a consequence of electron-ion correlations resulting in the temporary localization of some electrons near granular ions. Generally, the $q$ dependence of the curves in Fig. 6 is similar to that obtained in the case when a harmonic potential was applied (Fig. 3).

The intensities scattered by both bound and trapped electrons are shown in Fig. 7. The offset between the intensities $\langle\mathscr{I}(\boldsymbol{q})\rangle_{R}$ and $\mathscr{I}^{C}(\boldsymbol{q})$ at high $q$ is due to the trapped electron contribution $N_{t}$ and the inelastic scattering on the bound electrons. For the considered case even the intensity that neglects correlations between the trapped electrons, $\left\langle\mathscr{I}^{\text {uncorr }}(\boldsymbol{q})\right\rangle_{R}$, approximates well the full scattering signal. In the calculations presented, $N_{e}=0$.

Let us recall here that the structural information is carried by the elastically scattered photons. However, in currently planned experiments on nonperiodic single objects, inelastic scattering will also contribute to the measured patterns. Coherent XFEL sources have been reported to produce radiation with a bandwidth of $0.2-1 \%$ [2]. At a photon energy of $E_{\mathrm{ph}}=12 \mathrm{keV}$, it corresponds to a bandwidth of $\Delta E_{\mathrm{ph}}=20-120 \mathrm{eV}$, which is comparable to the Compton broadening. As a consequence, even if detecting exactly at the
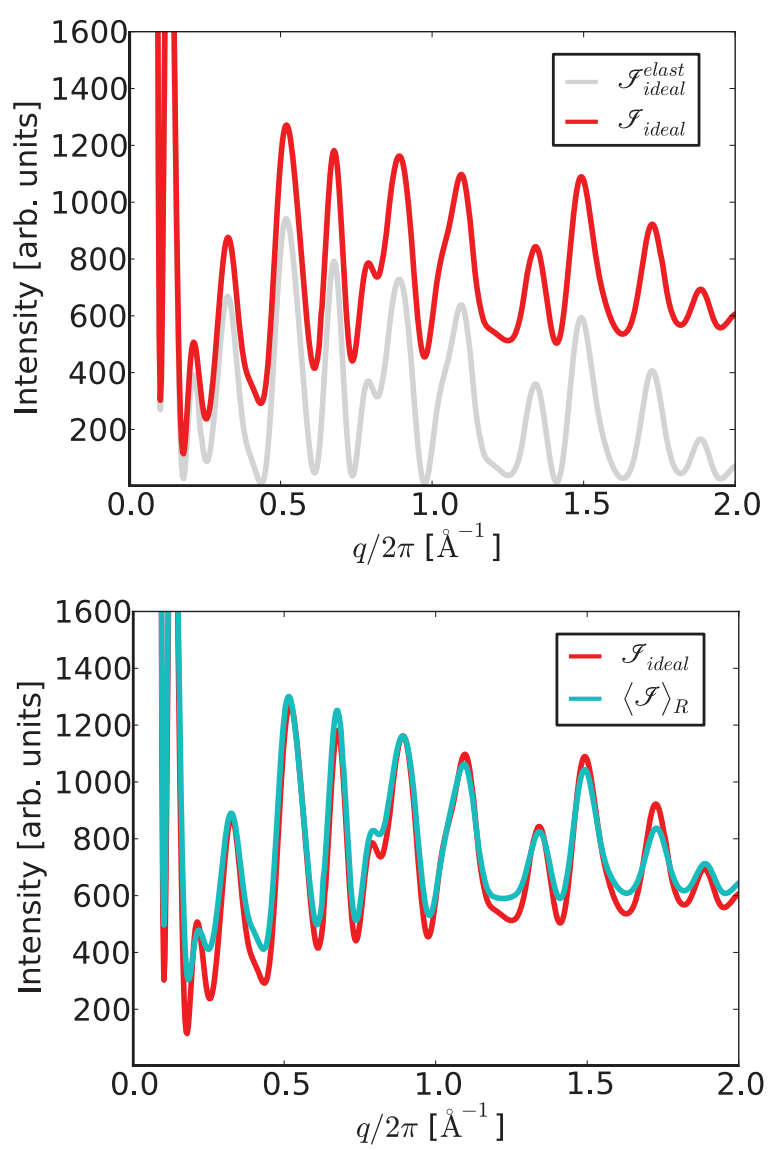

FIG. 8. (Color online) The effect of inelastic scattering and damage on the scattered intensity. We show: (a) the signal from the 'ideal' (undamaged) sample without inelastic scattering $\mathscr{I}_{\text {ideal }}^{\text {elast}}$, and with inelastic scattering $\mathscr{I}_{\text {ideal }}$, (b) the signal from the 'ideal' (undamaged) sample $\mathscr{I}_{\text {ideal }}$, and the signal from the damaged sample $\langle\mathscr{I}\rangle_{R}$, both including the inelastic scattering component. 
photon energy $E_{\mathrm{ph}}$, one collects the elastic scattering signal of incoming photons and inelastic scattering signal of incoming photons with the initial energy within the bandwidth. Both the elastic and the inelastic scattering then contribute to the measured signal.

We showed that inelastic scattering on bound electrons can have a significant impact on the measured intensities: it contributes to the background that reduces the contrast of the recorded image. This effect is even more pronounced at larger momentum transfers [ $\mathscr{I}_{b b \text {,ideal }}^{\text {elast }}$ and $\mathscr{I}_{b b \text {,ideal }}$ in Fig. 8(a)].

In experiments that have already been performed at LCLS, the effect of inelastic scattering was negligible. When the sample is a nanocrystal [32], the strong coherent Bragg peaks dominate over inelastic scattering. Inelastic scattering is also negligible at low resolution experiments on single objects [33], when intensity at small scattering vectors is collected. However, the above predictions show that the effect of inelastic scattering should be taken into account when planning atomic resolution imaging of nonperiodic samples.

Finally, we show the effect of damage on the recorded total signal [Fig. 8(b)]. As expected, progressing damage does not change the positions of intensity peaks that correspond to the positions of imaged ions. It only changes the (vertical) positions of intensity minima and maxima, which reduces the image contrast.

\section{SUMMARY}

The continuum approach is an efficient tool for the study of the dynamics of large atomic or molecular samples exposed to an intense XFEL pulse. However, the presently developed continuum models deliver only information on single-particle densities, and imaging studies require information on twoparticle correlations.

Here we have studied in detail the effect of two-particle correlations on CDI scattering patterns, obtained from electronion systems under conditions similar to those expected during XFEL imaging experiments at atomic resolution. We derived an estimate, Eq. (26), for the scattered intensities from the single-particle-density continuum model and demonstrated through numerical simulations that it can describe the scattered signal with good accuracy. Two-particle correlation effects manifest themselves only in the region of low $q$, together with the effects of the finite size of the sample. Self-correlations, due to the electron granularity, contribute to the equation by constant offsets. We also show that inelastic scattering on bound electrons can significantly contribute to the measured signal intensities: it contributes to the background that reduces the contrast of the recorded image. This effect is even more pronounced at larger momentum transfers. This implies that inelastic scattering can be neglected at experiments on nanocrystals, where Bragg scattering dominates the signal, and in experiments imaging single objects at low resolution. However, it should be taken into account when planning atomic resolution imaging of nonperiodic samples. Finally, we demonstrate how radiation damage influences the recorded total signal. We consider only the electronic damage, as the ionic damage caused by the change of ion positions due to the Coulomb interaction within the ionized system can be overcome by applying pulses that are short enough. The progressing damage does not change the positions of intensity peaks that correspond to the positions of imaged ions. It only changes the contrast between intensity minima and maxima, thus reducing the image contrast.

Our results are of significance for imaging-oriented studies of radiation damage performed with continuum models. They define the constraints for application of these models for CDI simulations.

\section{ACKNOWLEDGMENTS}

The authors would like to thank G. Dixit, C. Fortmann, S. P. Hau-Riege, and A. V. Martin for illuminating discussions.

\section{APPENDIX: SIMULATION DETAILS}

The simulations performed in this work were based on the molecular dynamics approach. The particles (ions and electrons) were treated as classical, charged-point-like particles.

\section{Ionization of atoms}

In order to describe the time dependence of the average number of bound electrons per atom, we used an approximate exponential form:

$$
N(t)=\left(N_{0}-N_{\text {inf }}\right) \exp (-w t)+N_{\text {inf }},
$$

where $N(t)$ denotes the number of bound electrons per atom at time $t$. Initially, at $t=0 \mathrm{fs}, N_{0}=6$. The fitting parameters, $w$ and $N_{\text {inf }}$, were adjusted to $N_{\text {inf }}=3.98$ and $w=0.456 \mathrm{fs}^{-1}$, following the results from Ref. [12]. Atomic form factors were calculated with the XATOM package $[34,35]$.

\section{Dynamics of trapped electrons}

The motion of the trapped electrons was followed with the Newton equations solved by the numerical velocity-Verlet algorithm. The interaction between the charged particles was described by the regularized Coulomb potential, $V(r)=$ $1 / \sqrt{r^{2}+r_{0}^{2}}$, where the cutoff parameter, $r_{0}=0.1 \AA$, was used. The simulation time step was 1 as.

Simulating the smooth positive background field for trapped electrons, we applied an external radial harmonic potential of the form $V(r)=-D r^{2} / 2$. Initial electron positions corresponded to those ones within an electron system in thermal equilibrium.

Simulating the case with granular ions, we placed the ions at random positions within a sphere of $30-\AA$ radius. The ion density was set to $1 / 15 \AA^{-3}$, which corresponds to a typical atomic density within a protein. The minimal atom-atom distance was kept larger than $1.5 \AA$. Ionic charge was set to +2 . Let us note that the static electronic charge was fully neutralized by ions inside a sphere of 7.1- $\AA$ radius.

\section{Calculation of scattered intensities}

We recorded the total number of the bound electrons at each atom or ion at a time step. We did not distinguish between 
bound electrons at different atomic orbitals. Therefore, we approximated the scattering factor of an ion with an average scattering factor, averaged over different electronic configurations. The scattering factor of a trapped electron was 1 .
In our simulation, we considered diffractive scattering only from ions inside the neutralizing 7.1- $\AA$-radius sphere (corresponding to the imaged net-neutral electron-ion sample) and not from the ions in the positively charged outer shell.
[1] R. Neutze, R. Wouts, D. van der Spoel, E. Weckert, and J. Hajdu, Nature (London) 406, 752 (2000).

[2] P. Emma, R. Akre, J. Arthur, R. Bionta, C. Bostedt, J. Bozek, A. Brachmann, P. Bucksbaum, R. Coffee, F.-J. Decker et al., Nat. Photonics 4, 641 (2010).

[3] D. Pile, Nat. Photonics 5, 456 (2011).

[4] M. Altarelli, R. Brinkmann, M. Chergui, W. Decking, B. Dobson, S. Düsterer, G. Grübel, W. Graeff, H. Graafsma, J. Hajdu et al., The European X-Ray Free-Electron Laser, Technical design report, DESY 2006-097 (2007).

[5] G. Huldt, A. Szoke, and J. Hajdu, J. Struct. Biol. 144, 219 (2003).

[6] G. Bortel and G. Faigel, J. Struct. Biol. 158, 10 (2007).

[7] V. L. Shneerson, A. Ourmazd, and D. K. Saldin, Acta Crystallogr., Sect. A 64, 303 (2008).

[8] N.-T. D. Loh and V. Elser, Phys. Rev. E 80, 026705 (2009).

[9] G. Bortel, G. Faigel, and M. Tegze, J. Struct. Biol. 166, 226 (2009).

[10] R. Fung, V. Shneerson, D. K. Saldin, and A. Ourmazd, Nat. Phys. 5, 64 (2009).

[11] B. Ziaja, A. R. B. de Castro, E. Weckert, and T. Möller, Eur. Phys. J. D 40, 465 (2006).

[12] S. P. Hau-Riege, R. A. London, and A. Szoke, Phys. Rev. E 69, 051906 (2004)

[13] R. W. James, The Optical Principles of the Diffraction of X-Rays (G. Bell, London, 1962).

[14] J. H. Hubbell, Wm. J. Veigele, E. A. Briggs, R. T. Brown, D. T. Cromer, and R. J. Howerton J. Phys. Chem. Ref. Data 4, 471 (1975).

[15] G. Dixit, O. Vendrell, and R. Santra, PNAS 109, 11636 (2012).

[16] Z. Jurek, G. Faigel, and M. Tegze, Eur. Phys. J. D 29, 217 (2004).

[17] C. Gnodtke, U. Saalmann, and J.-M. Rost, New J. Phys 13, 013028 (2011).

[18] R. Santra, J. Phys. B 42, 023001 (2009).
[19] B. Ziaja, D. van der Spoel, A. Szöke, and J. Hajdu, Phys. Rev. B 64, 214104 (2001).

[20] B. Ziaja, A. Szöke, D. van der Spoel, and J. Hajdu, Phys. Rev. B 66, 024116 (2002).

[21] S. Glenzer and R. Redmer, Rev. Mod. Phys. 81, 1625 (2009).

[22] M. S. Murillo and J. C. Weisheit, Phys. Rep. 302, 1 (1998).

[23] Z. Jurek and G. Faigel, Eur. Phys. J. D 50, 35 (2008).

[24] J. Chihara, J. Phys. F 17, 295 (1987).

[25] J. Chihara, J. Phys. Condens. Matter 12, 231 (2000).

[26] E. Nardi, Phys. Rev. A 43, 1977 (1991).

[27] G. Gregori, S. H. Glenzer, W. Rozmus, R. W. Lee, and O. L. Landen, Phys. Rev. E 67, 026412 (2003).

[28] A. Höll, T. Bornath, L. Cao, T. Döppner, S. Düsterer, E. Förster, C. Fortmann, S. H. Glenzer, G. Gregori, T. Laarmann et al., High Energy Density Phys. 3, 120 (2007).

[29] A. Authier, Dynamical Theory of X-Ray Diffraction (Oxford University Press, Oxford, 2001).

[30] Y. Ding, A. Brachmann, F.-J. Decker, D. Dowell, P. Emma, J. Frisch, S. Gilevich, G. Hays, P. Hering, Z. Huang et al., Phys. Rev. Lett. 102, 254801 (2009).

[31] J.-P. Hansen and I. R. McDonald, Theory of Simple Liquids (Academic, Amsterdam, 2006).

[32] H. N. Chapman, P. Fromme, A. Barty, T. A. White, R. A. Kirian, A. Aquila, M. S. Hunter, J. Schulz, D. P. DePonte, U. Weierstall et al., Nature (London) 470, 73 (2011).

[33] M. M. Seibert, T. Ekeberg, F. R. N. C. Maia, M. Svenda, J. Andreasson, O. Jönsson, D. Odić, B. Iwan, A. Rocker, D. Westphal et al., Nature (London) 470, 78 (2011).

[34] S.-K. Son, L. Young, and R. Santra, Phys. Rev. A 83, 033402 (2011).

[35] S.-K. Son and R. Santra, XАTOM, an integrated toolkit for $\mathrm{X}$-ray and atomic physics, revision 390, CFEL, DESY, Hamburg, Germany, 2011. 